\title{
Factores psicosociales y hábitos asociados con dispepsia funcional en internos de un hospital nacional en Piura, Perú
}

\section{Psychosocial Factors and Habits Associated with Functional Dispepsy in Intern at a National Hospital in Piura-Peru}

Christian R. Mejia, MD, ${ }^{1}$ Claudia Quezada-Osoria, MD, ${ }^{2}$ Araseli Verastegui-Díaz, MS, ${ }^{3}$ Matlin M. Cárdenas, MS, ${ }^{4}$

Katerine M. García-Moreno, MS, ${ }^{5}$ Dante M. Quiñones-Laveriano, MS. ${ }^{6}$

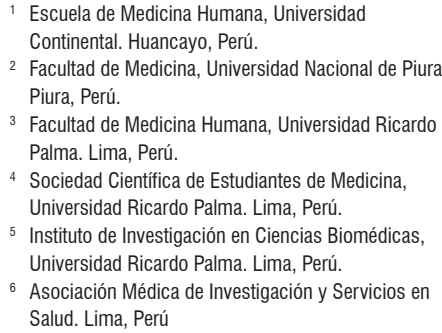

2 Facultad de Medicina, Universidad Nacional de Piura. Piura, Perú.

3 Facultad de Medicina Humana, Universidad Ricardo Palma. Lima, Perú.

4 Sociedad Científica de Estudiantes de Medicina, Universidad Ricardo Palma. Lima, Perú.

${ }^{5}$ Instituto de Investigación en Ciencias Biomédicas, Universidad Ricardo Palma. Lima, Perú.

Asociación Médica de Investigación y Servicios en Salud. Lima, Perú

\begin{abstract}
Resumen
Objetivos: determinar los factores psicosociales y hábitos asociados con dispepsia funcional (DF) en internos de medicina en el Hospital de Apoyo II-2 de Sullana, Piura. Métodos: estudio longitudinal prospectivo desarrollado en 24 internos de medicina. Se tomaron 5 encuestas, 1 basal antes de iniciar el internado médico y al finalizar cada rotación. Se usó la prueba (o test) "A new questionnaire for the diagnosis of dyspepsia" para el diagnóstico de DF; además, todas las pruebas utilizadas fueron validadas. Para el análisis bivariado y multivariado, se utilizó el PA-GEE, usando la familia binomial y función de enlace log, obteniendo riesgos relativos crudos (RRc) y ajustados (RRa) y sus intervalos de confianza al 95\%. Resultados: la edad promedio fue de 24,1 años $( \pm 1,4)$; el $58 \%$ (14) fueron mujeres. La prevalencia de dispepsia en el internado fue del $32 \%$. El tener ansiedad ( $p<0,001$; RRa: 5,9; IC 95\%: 2,88-12,13) o depresión ( $p=0,015 ; \mathrm{RRa:} 3,4 ;$ IC 95\%: $1,27-9,29)$ fueron factores de riesgo para padecer DF; el obtener una nota mejor al finalizar las rotaciones fue un factor protector ( $p=0,020$; RRa: 0,8; IC 95\%: 0,71-0,97), ajustado por tener irritabilidad, y el consumo de bebidas energizantes. Conclusiones: según los datos evaluados, aquellos que cursen con algún trastorno psicosocial durante el internado están predispuestos a sufrir mayores riesgos para enfermedades gastrointestinales, por lo que las instituciones deben tener programas de detección y vigilancia de estas patologías.
\end{abstract}

\section{Palabras clave}

Dispepsia, estudiantes de medicina, depresión, ansiedad.

\begin{abstract}
Objectives: The objective of this study was to determine psychosocial factors and habits associated with functional dyspepsia (FD) in medical interns at the Sullana-Piura II-2 support hospital. Methods: This was a prospective longitudinal study of 24 medical interns who answered five surveys: one baseline survey before beginning their medical internships, and another survey at the end of each rotation. The test "A new questionnaire for the diagnosis of dyspepsia" was used for diagnosis of FD, and all the tests used were validated. For bivariate and multivariate analysis, population averaged generalized estimating equations (PA-GEE) were used, using the binomial family and log link function, to obtain raw relative risks (rRR) and adjusted relative risks (aRR) and their 95\% confidence intervals. Results: The mean age was 24.1 years $(+/-1.4), 58 \%$ (14) were women. The prevalence of dyspepsia among interns was $32 \%$. Having anxiety ( $p<0.001$, aRR: $5.9,95 \%$ $\mathrm{Cl}: 2.88-12.13$ ) or depression ( $p=0.015$, aRR: $3.4,95 \% \mathrm{Cl}: 1.27-9.29$ ) were risk factors for FD, obtaining a better score at the end of a rotation was a protective factor ( $p=0.020$, aRR: $0.8,95 \% \mathrm{Cl}: 0.71-0.97)$. RRs were adjusted for irritability and consumption of energy drinks. Conclusions: According to the data evaluated, those who have a psycho-social disorder during hospitalization are predisposed to suffer greater risks for gastro-intestinal diseases, so that institutions must have programs to detect and monitor these pathologies.
\end{abstract}

\section{Keywords}

Dyspepsia, medical students, depression, anxiety, (Source: DeCS BIREME). 


\section{INTRODUCCIÓN}

La dispepsia funcional (DF) se caracteriza por la presencia en el último trimestre de cuatro síntomas cardinales: plenitud posprandial, saciedad temprana, dolor epigástrico y ardor epigástrico. Es uno de los trastornos gastrointestinales más comunes en la población (1-3), ya que, a nivel mundial, la dispepsia puede llegar a constituir hasta el $40 \%$ de las patologías gastrointestinales, pero solo la décima parte de este grupo de pacientes buscan atención médica $(4,5)$, lo que dificulta aún más su diagnóstico por el hecho de que no existe un órgano específico que sea afectado (6, 7 ). Generalmente es detectada por la presencia de los grupos más frecuentes de molestias: el síndrome de dolor en el epigastrio y el síndrome de distrés posprandial (8).

Son escasos los reportes de dispepsia en adultos jóvenes, a pesar de que estos son los grupos más afectados (9) por su relación con los trastornos dietéticos y por la alta prevalencia de patologías psicosociales asociadas (10-13). En cuanto a este grupo etario, el sector estudiantil tiene mucho riesgo por la sobrecarga académica, la ansiedad $(14,15)$, la depresión $(10,16)$, la irritabilidad y el estrés -este último el más abordado- $(1,12)$. De ahí la importancia de su estudio en poblaciones que tienen mayores niveles de estrés (17), como lo son los estudiantes de ciencias biomédicas, ya que sufren de una constante y creciente exigencia, sobrecarga académica, evaluaciones y la inadecuada gestión del tiempo (18). Esto se incrementa en el último año de medicina, ya que se le suma la responsabilidad laboral, las largas jornadas de atención, entre otros (19).

Por tanto, nuestro objetivo fue determinar los factores psicosociales y los hábitos asociados con DF en internos de medicina en el Hospital de Apoyo II-2 de Sullana, Piura.

\section{MATERIAL Y MÉTODO}

\section{Diseño de estudio}

Se realizó un estudio de tipo longitudinal prospectivo desde diciembre de 2011 hasta diciembre de 2012. En los internos de medicina del Hospital de Apoyo II-2 de Sullana, Piura, se llevaron a cabo encuestas autoaplicadas, una antes de comenzar el internado médico y las otras después de finalizar cada una de las cuatro rotaciones. Se incluyó a la totalidad de internos que realizó el internado en dicho hospital y que aceptaron participar voluntariamente en el estudio; se excluyeron dos mediciones de un interno (la medición basal y la ulterior a su primera rotación), que ya habían sido realizadas en un hospital de la seguridad social de Piura. Así mismo, se excluyó la última medición de una interna, ya que entregó su encuesta a destiempo.

\section{Instrumentos y variables}

Se realizó un cuestionario conformado por preguntas abiertas y cerradas. La variable dependiente fue elaborada a partir de las respuestas que dieron al cuestionario para determinar dispepsia, según la escala validada en español para el diagnóstico de dispepsia, "A new questionnaire for the diagnosis of dyspepsia”. El cuestionario fue considerado positivo si habían 3 o más puntos de los 9 ítems de la encuesta.

Los otros factores evaluados fueron los socioeconómicos (edad, género, estado civil, paternidad e independencia económica); los datos académicos (año de ingreso, semestre académico en curso, si ha desaprobado un curso, horas de estudio fuera del horario de clases de la universidad); las medidas antropométricas y los antecedentes clínicos IMC (según los criterios de la Organización Mundial de la Salud [OMS]); los hábitos alimenticios (cumplimiento del horario de alimentación, lugar donde se alimenta); la ansiedad (escala validada en español para el diagnóstico de ansiedad de Zung, positivo si se obtenía $\geq 56 / 80$ puntos); la depresión (escala validada en español para el diagnóstico de depresión de Beck, positivo para depresión leve si se obtenían 4-8 de 20 puntos); la irritabilidad (escala de Minnesota Multiphasic Personality Inventory-2, positivo si se obtenían más de 5 puntos de los 16); el hábito tabáquico (escala validada en español de Fargerstrom, positivo si se obtenían 4 de 10 puntos); el hábito alcohólico (escala de CAGE, positivo si se obtenían 2 de 4 puntos); y el consumo de bebidas energizantes.

\section{Procedimiento}

Se confeccionó una encuesta con base en las pruebas validadas. Luego se sometió al Comité de Ética e Instancias Administrativas del Hospital de Apoyo II-2 de Sullana y de la Universidad Nacional de Piura (UNP). Los resultados preliminares fueron presentados como parte de la tesis de pregrado de uno de los autores (CQO).

La primera encuesta se tomó en diciembre de 2011, días antes de que los internos empezaran sus rotaciones en el Hospital. La segunda, tercera, cuarta y quinta medición fueron tomadas al finalizar cada una de las rotaciones: cirugía, ginecología, pediatría y medicina interna. Una vez obtenidos los datos, se los pasó a una base generada en el programa MS Excel para Windows 2010. Por último, se procedió a la depuración de los datos previo al análisis estadístico.

\section{Análisis estadístico}

Los datos fueron analizados en el paquete estadístico Stata 11,1. En el análisis descriptivo se evaluaron las variables 
numéricas con la prueba estadística Shapiro Wilk, para determinar su normalidad, describiéndolas mediante las medias y la desviación estándar. Se describieron las variables categóricas utilizando frecuencias y porcentajes. Para la estadística analítica longitudinal se utilizó la prueba estadística PA-GEE, se obtuvieron los riesgos relativos crudos (RRc) y ajustados (RRa), así como sus intervalos de confianza al 95\% (IC 95\%). Para esto, se usó la familia binomial, la función de enlace log y con modelos robustos. La variable de tiempo fue la rotación en la que se tomó el cuestionario. Se consideró como estadísticamente significativo un valor de $\mathrm{p}<0,05$.

\section{RESULTADOS}

Se encuestó a la totalidad de los 24 internos que rotaron en el Hospital de Apoyo II-2 de Sullana durante el 2012. La edad promedio de los participantes fue de 24,1 años (desviación estándar [DE]: 1,39 años). El 58,3\% (14) fueron mujeres; el $100 \%$ eran solteros; solo 1 (4,2\%) tenía un hijo y el 50,0\% (12) vivían solos; el 54,2\% (13) de los internos provenía de la Universidad Nacional de Piura. Las características socioacadémicas de los encuestados se muestran en la tabla 1 .

En la tabla 2 se muestran las características agrupadas por el trimestre donde fue tomada la encuesta. El 28,2\% de los internos tuvo dispepsia funcional durante las 5 mediciones, encontrando los porcentajes más altos en el primer y tercer trimestre $(23,5 \%)$.

La dependencia de alcohol basal fue del 8,4\% (4), porcentaje que se fue incrementando paulatinamente hasta los porcentajes máximos de $27,7 \%$ (13) encontrados en el 3 . $^{\text {er }}$ y 4 . $^{\circ}$ trimestre. Lo mismo sucedió con el reporte de problemas para dormir, que fue del $0 \%(0)$ en la medición basal y que se incrementó paulatinamente hasta el 28,1\% (16) reportado en la última medición (tabla 2).

Asimismo, se estudió la presentación de nuestra variable principal y de los factores, según las rotaciones. Dichos resultados se muestran en la tabla 3. En relación con la dispepsia, se observó un mayor número en la rotación de cirugía $(15 ; 50 \%)$, seguida por medicina interna $(6 ; 20 \%)$, pediatría $(5 ; 16,7 \%)$ y ginecología $(4 ; 13,3 \%)$. Con respecto a los factores psicosociales, los internos que rotaron por cirugía fueron los que tuvieron mayor ansiedad, depresión e irritabilidad con 7 (41,2\%), 5(38,5\%) y $12(38,5 \%)$, respectivamente.

En cuanto a las drogas legales, el consumo de alcohol fue mayor cuando los internos rotaron en Cirugía y Ginecología (27,9\%); el consumo de café fue mayor en la rotación de Pediatría (29,2\%); y en la rotación de Cirugía hubo mayores problemas del sueño $(28,1 \%)$ (tabla 3 ).

En el análisis bivariado se encontró una fuerte asociación entre la dispepsia y la depresión $(\mathrm{p}<0,001)$, la ansiedad ( $\mathrm{p}$
Tabla 1. Características socioacadémica de los internos de medicina del Hospital de Apoyo II-2 de Sullana, 2012

\begin{tabular}{lcc}
\hline \multicolumn{1}{c}{ Variables } & $\mathbf{n}$ & $\mathbf{( \% )}$ \\
\hline Género & & \\
\hline Masculino & 10 & 41,7 \\
Femenino & 14 & 58,3 \\
\hline Edad & 24,1 & $\pm 1,39$ \\
\hline Lugar de nacimiento & & \\
\hline Piura & 11 & 45,8 \\
Sullana & 5 & 20,8 \\
Trujillo & 3 & 12,5 \\
Otros & 5 & 20,9 \\
\hline Universidad de procedencia & & \\
\hline UNP & 13 & 54,2 \\
UPAO-Trujillo & 9 & 37,5 \\
UCV-Trujillo & 2 & 8,3 \\
\hline Convivencia & & \\
\hline Solo & 12 & 50,0 \\
Padres y/o hermanos & 6 & 25,0 \\
Pareja & 4 & 16,7 \\
Amigos & 2 & 8,3 \\
\hline Solventa gastos & & \\
\hline Trabaja & 2 & 8,3 \\
Dinero de padres & 2 & 8,3 \\
Trabaja y recibe dinero & 9 & 33,3 \\
\hline Horas por día que estudia & 20 & 83,4 \\
\hline Menos de 1 hora & & \\
1-3 horas & & \\
3-5 horas & & \\
\hline & & \\
\hline
\end{tabular}

UCV: Universidad César Vallejo; UNP: Universidad Nacional de Piura; UPAO: Universidad Privada Antenor Orrego.

${ }^{*}$ Mediana y desviación estándar.

$<0,001)$, la nota obtenida en la rotación $(p=0,014)$, la irritabilidad $(p=0,007)$ y el consumo de bebidas energizantes $(\mathrm{p}=0,019)($ tabla 4$)$.

En el análisis multivariado, el riesgo de dispepsia se incrementó por tener asociado un grado de depresión ( $p$ = 0,015; RRa: 3,4; IC 95\%: 1,27-9,29) o de ansiedad ( $\mathrm{p}$ $<0,001$; RRa: 5,9; IC 95\%: 2,88-12,13). En cambio, el riesgo de dispepsia disminuyó frente a una mayor nota como resultado de su rotación ( $p=0,020$; RRa: 0,8 ; IC 95\%: 0,71-0,97). Siendo estos ajustados por la irritabilidad y el consumo de bebidas energizantes (tabla 4).

\section{DISCUSIÓN}

Es importante reconocer los factores que influyen en la aparición y desarrollo de la DF, debido a la alta prevalencia y por no existir una causa orgánica a tratar, sobre todo 
Tabla 2. Frecuencia de características asociadas con dispepsia según el trimestre en que se tomó la medición

\begin{tabular}{|c|c|c|c|c|c|c|c|c|c|c|}
\hline \multirow[b]{3}{*}{ Dispepsia funcional } & \multicolumn{10}{|c|}{ Trimestre en que se tomó la medición $\mathrm{n}(\%)$} \\
\hline & \multicolumn{2}{|c|}{ Basal } & \multicolumn{2}{|c|}{ 1. er } & \multicolumn{2}{|c|}{$2 .{ }^{\circ}$} & \multicolumn{2}{|c|}{3 er $^{\text {er }}$} & \multicolumn{2}{|c|}{$4 .^{\circ}$} \\
\hline & 3 & $(8,8)$ & 8 & $(23,5)$ & 9 & $(26,5)$ & 8 & $(23,5)$ & 5 & $(17,7)$ \\
\hline Dispepsia estómago lleno & 2 & $(11,0)$ & 5 & $(27,8)$ & 5 & $(27,8)$ & 3 & $(16,7)$ & 3 & $(16,7)$ \\
\hline Dispepsia estómago vacío & 1 & $(7,1)$ & 3 & $(21,4)$ & 4 & $(28,6)$ & 4 & $(28,6)$ & 2 & $(14,3)$ \\
\hline Dispepsia estómago mixto & 0 & $(0)$ & 0 & $(0)$ & 0 & $(0)$ & 1 & $(100)$ & 0 & $(0)$ \\
\hline Ansiedad & 1 & $(5,6)$ & 3 & $(16,7)$ & 6 & $(33,3)$ & 6 & $(33,3)$ & 2 & $(11,1)$ \\
\hline Depresión & 1 & $(7,1)$ & 4 & $(28,6)$ & 4 & $(28,6)$ & 4 & $(28,6)$ & 1 & $(7,1)$ \\
\hline Irritabilidad & 2 & $(6,1)$ & 7 & $(21,2)$ & 11 & $(33,3)$ & 8 & $(24,2)$ & 5 & $(15,2)$ \\
\hline Tabaco & 6 & $(22,2)$ & 5 & $(18,5)$ & 5 & $(18,5)$ & 6 & $(22,2)$ & 5 & $(18,5)$ \\
\hline Alcohol & 4 & $(8,4)$ & 7 & $(14,9)$ & 10 & $(21,3)$ & 13 & $(27,7)$ & 13 & $(27,7)$ \\
\hline Café & 2 & $(7,6)$ & 6 & $(23,1)$ & 6 & $(23,1)$ & 6 & $(23,1)$ & 6 & $(23,1)$ \\
\hline Bebidas energizaste & 3 & $(8,2)$ & 11 & $(29,7)$ & 4 & $(10,8)$ & 8 & $(21,6)$ & 11 & $(29,7)$ \\
\hline Problemas para dormir & 0 & $(0)$ & 12 & $(21,1)$ & 14 & $(24,5)$ & 15 & $(26,3)$ & 16 & $(28,1)$ \\
\hline Cumple horarios de comida & 23 & $(31,9)$ & 12 & $(16,7)$ & 14 & $(19,4)$ & 13 & $(18,1)$ & 10 & $(13,9)$ \\
\hline \multicolumn{11}{|l|}{ Lugar donde se alimentan } \\
\hline Pensión & 18 & $(19,6)$ & 18 & $(19,6)$ & 19 & $(20,6)$ & 19 & $(20,6)$ & 18 & $(19,6)$ \\
\hline Casa & 5 & $(20)$ & 5 & $(20)$ & 5 & $(20)$ & 5 & $(20)$ & 5 & $(20)$ \\
\hline
\end{tabular}

Tabla 3. Frecuencia de características asociadas con dispepsia según las rotaciones que tuvieron

\begin{tabular}{lcccccccc}
\hline & \multicolumn{9}{c}{ Rotaciones $\mathbf{n}(\%)$} \\
\cline { 2 - 9 } & \multicolumn{2}{c}{ Cirugía } & \multicolumn{2}{c}{ Ginecología } & \multicolumn{2}{c}{ Pediatría } & \multicolumn{2}{c}{ Medicina } \\
\hline Dispepsia & 15 & $(50,0)$ & 4 & $(13,3)$ & 5 & $(16,7)$ & 6 & $(20,0)$ \\
Dispepsia estómago lleno & 8 & $(50,0)$ & 3 & $(18,8)$ & 3 & $(18,8)$ & 2 & $(12,5)$ \\
Dispepsia estómago vacío & 6 & $(46,2)$ & 1 & $(7,7)$ & 2 & $(15,4)$ & 4 & $(30,8)$ \\
Dispepsia estómago mixto & 1 & $(100)$ & 0 & $(0)$ & 0 & $(0)$ & 0 & $(0)$ \\
Ansiedad & 7 & $(41,2)$ & 2 & $(11,8)$ & 2 & $(11,8)$ & 6 & $(35,3)$ \\
Depresión & 5 & $(38,5)$ & 2 & $(15,4)$ & 5 & $(38,5)$ & 1 & $(7,7)$ \\
Irritabilidad & 12 & $(38,5)$ & 2 & $(15,4)$ & 5 & $(38,5)$ & 1 & $(7,7)$ \\
Tabaco & 5 & $(23,8)$ & 6 & $(28,6)$ & 5 & $(23,8)$ & 5 & $(23,8)$ \\
Alcohol & 12 & $(27,9)$ & 12 & $(27,9)$ & 11 & $(25,6)$ & 8 & $(18,6)$ \\
Café & 6 & $(25,0)$ & 5 & $(20,8)$ & 7 & $(29,2)$ & 6 & $(25,0)$ \\
Bebidas energizantes & 9 & $(26,5)$ & 10 & $(29,4)$ & 9 & $(26,5)$ & 6 & $(17,7)$ \\
\hline
\end{tabular}

Tabla 4. Análisis bi y multivariado longitudinal de dispepsia funcional y su asociación con variables en internos de medicina del Hospital de Apoyo II-2 de Sullana; 2012

\begin{tabular}{lcccc}
\hline & \multicolumn{2}{c}{ Bivariado } & \multicolumn{2}{c}{ Multivariado* } \\
\cline { 2 - 5 } & RRc (IC 95\%) & $\mathbf{p}$ & RRa (IC 95\%) & $\mathbf{p}$ \\
\hline Depresión & $2,7(1,56-4,54)$ & $<0,001$ & $3,4(1,27-9,29)$ & 0,015 \\
Ansiedad & $4,6(2,66-7,88)$ & $<0,001$ & $5,9(2,88-12,13)$ & $<0,001$ \\
Nota obtenida en la rotación & $0,8(0,74-0,97)$ & 0,014 & $0,8(0,71-0,97)$ & 0,020 \\
Irritabilidad & $2,0(1,22-3,49)$ & 0,007 & $0,9(0,55-1,40)$ & 0,587 \\
Consumo de bebidas energizantes & $2,3(1,15-4,52)$ & 0,019 & $1,5(0,84-2,75)$ & 0,171 \\
\hline
\end{tabular}

IC 95\%: intervalos de confianza del 95\%; p: valor p; RRa: riesgos relativos ajustados; RRc: riesgos relativos crudos; obtenidos usando PA-GEE con familia binomial, función de enlace log y modelos robustos, variable tiempo la rotación en la que se tomó el cuestionario. 
en grupos en riesgo para esta patología. La prevalencia de dispepsia funcional encontrada en los internos fue mayor en el primer y tercer trimestre. Asimismo, se observó una mayor frecuencia en determinadas rotaciones (Cirugía); esto puede deberse a que en dicho hospital, esa rotación involucra una gran responsabilidad y los médicos asistentes son bastante exigentes, lo que representaba estar una mayor cantidad de horas en el hospital -a comparación de otras rotaciones- $y$ tener que cumplir un récord mínimo de procedimientos quirúrgicos. Esto concuerda con un estudio realizado en una universidad privada de la capital, en la que se encontró que la alta presión académica, la sobrecarga laboral, la inestabilidad financiera, la falta de sueño y la cercanía con la enfermedad y las dolencias del paciente tenían un efecto negativo en la salud de los estudiantes, encontrándose en este grupo mayor prevalencia de psicopatología, depresión, ansiedad, abuso de sustancias e ideación suicida (20).

La DF se asoció con el tener algún grado de depresión, lo que representó 1,4 veces más riesgo de tener dispepsia a comparación de los que no tuvieron algún grado de depresión. Lo anterior concuerda con un estudio realizado por Silva y colaboradores, quienes encontraron que los pacientes con depresión mostraron 3 veces más riesgo de tener dispepsia funcional (21). Esto también concuerda con un estudio realizado en cuatro facultades de medicina en Latinoamérica, en donde la depresión es un factor que se asocia con el tener DF (22). Por tanto, esta patología debería ser vigilada y controlada en estos grupos en riesgo, sobre todo por las posibles consecuencias a corto y largo plazo que pudiese generar.

Otra fuerte asociación encontrada fue que aquellos que tuvieron ansiedad tenían 3,9 veces más riesgo de padecer dispepsia, lo que concuerda con lo estudiado por Molano y colaboradores, quienes encontraron que el 50,1\% de los adolescentes con dispepsia tenía síntomas de ansiedad y el 53,6\% tenía síntomas de depresión. Asimismo, Mak y colaboradores observaron una mayor prevalencia de dispepsia entre aquellos que tenían ansiedad en la categoría de "Alto" (20,3\%) que entre los que no fueron dispépticos $(10,8 \%)(10)$.

Se encontró que el tener una mejor nota al finalizar la rotación fue un factor protector para no sufrir de dispepsia. Esto concuerda con un estudio que concluyó que el $61,1 \%$ de jóvenes con dispepsia atribuía la aparición de sus síntomas a preocupaciones de origen académico, seguido por dificultades familiares (23). Otro estudio que refuerza este hallazgo es uno realizado en ocho facultades de medicina del Perú, en donde el desaprobar un curso incrementó la frecuencia de padecer dispepsia (razón de prevalencias: 1,24; intervalo de confianza: 1,13-1,37) (24). Este aspecto debería profundizarse a futuro, ya que una baja calificación podría provocar frustración y estrés por no lograr el objetivo deseado, lo que podría estar repercutiendo negativamente en los estudiantes. Próximos estudios deberían abordar este tema, además de posibles intervenciones para mejorar esta situación.

Otro dato relevante fue el aumento progresivo del consumo de alcohol conforme fueron pasando los trimestres, que llegó a incrementarse más de dos veces de la medición basal. Esto es acorde con los diversos reportes en los que el consumo de cigarrillos y alcohol fue significativo en los adolescentes con dispepsia (15). Una revisión en Chile mostró que el $80 \%$ de los internos consumía alcohol y que el 6,4\% de los estudiantes de medicina mostró dependencia alcohólica. Estos datos son alarmantes debido a que su excesivo consumo incrementa el riesgo de aparición de muchos problemas de salud, Si esto no es controlado, puede mantenerse y afectar seriamente a esta población cuando llegue a ser profesional (3).

En cuanto a las limitaciones del estudio, tuvimos una muestra pequeña. Por esta razón, los resultados no se pueden extrapolar a la totalidad de internos de medicina del Perú, pero aun así los datos son importantes porque no existen reportes previos de seguimiento a este tipo de poblaciones en nuestro medio, además de ser un estudio que contó con estudiantes de medicina de universidades públicas y privadas, que se asemejan a otros grupos poblacionales. Otra limitante fue que por tratarse de un estudio censal, los intervalos de confianza no fueron la mejor medida estadística, aunque sirven para poder comparar los resultados con otros estudios. Se recomienda llevar a cabo este estudio en poblaciones más grandes y con un muestreo adecuado, para encontrar relaciones que puedan extrapolarse.

Con base en la población estudiada, se concluye que los internos con algún grado de depresión, ansiedad y dependencia alcohólica tienen mayor riesgo de presentar dispepsia funcional. El tener una mejor nota al finalizar la rotación es un factor protector para la DF.

\section{Agradecimientos}

Se agradece el aporte previo que hicieron los alumnos Fiorella Vilca y Stefan Carrasco para la mejora previa del manuscrito.

\section{Financiación}

Autofinanciado.

\section{Presentaciones previas}

La médica Claudia Quezada Osoria presentó algunos resultados previos del trabajo como parte de su tesis para el título de médico-cirujano. 


\section{REFERENCIAS}

1. Basandra S, Bajaj D. Epidemiology of dyspepsia and irritable bowel syndrome (IBS) in medical students of northern india. J Clin Diagn Res JCDR. 2014;8(12):JC13-6.

2. Mahadeva S, Goh KL. Epidemiology of functional dyspepsia: a global perspective. World J Gastroenterol. 2006; 12(17):2661-6.

3. Brun R, Kuo B. Review: functional dyspepsia. Ther Adv Gastroenterol. 2010;3(3):145-64.

4. Moser G. Funktionelle gastrointestinale Störungen. Wien Med Wochenschr. 2006;156(15-16):435-40.

5. David A. McClellan RAL. Update on the evaluation and management of functional dyspepsia. Am Acad Fam Physicians. 2011;83(5):547-52.

6. Longstreth GF. Functional dyspepsia - managing the conundrum. N Engl J Med. 2006;354(8):791-3.

7. Zolezzi Francis A. Las enfermedades funcionales gastrointestinales y Roma III. Rev Gastroenterol Perú. 2007;27(2):177-84.

8. Mearin F, Calleja JL. Definiendo la dispepsia funcional. Rev Esp Enferm Dig. 2011;103(12):640-7.

9. Grover S, Kumar V, Chakrabarti S, et al. Prevalence and type of functional somatic complaints in patients with first-episode depression. East Asian Arch Psychiatry. 2012;22(4):146-53.

10. Mak ADP, Wu JCY, Chan Y, et al. Dyspepsia is strongly associated with major depression and generalised anxiety disorder - a community study. Aliment Pharmacol Ther. 2012;36(8):800-10.

11. Van Oudenhove L, Tack J. New epidemiologic evidence on functional dyspepsia subgroups and their relationship to psychosocial dysfunction. Gastroenterology. 2009;137(1):23-6.

12. Tobón $S$, Núñez A. Relación de factores psicológicos con los síntomas de dispepsia en estudiantes universitarios españoles. Suma Psicológica. 2007;14(1):93-106.

13. Levy RL, Olden KW, Naliboff BD, et al. Psychosocial aspects of the functional gastrointestinal disorders. Gastroenterology. 2006;130(5):1447-58.

14. Aro P, Talley NJ, Ronkainen J, et al. Anxiety is associated with uninvestigated and functional dyspepsia (Rome III criteria) in a Swedish population-based study. Gastroenterology. 2009; 137(1):94-100.

15. Molano J, Piñeros S, López de Mesa C. The relation of dyspepsia and gastroesophageal reflux to emotional factors: impact on the education and social activity of adolescents. Rev Colomb Gastroenterol. 2009;24(4):364-72.

16. Álvarez OM, Ulloa B, Fernández J, et al. Afecciones digestivas más frecuentes en el adulto mayor. MEDISAN. 2010;14(4):511.

17. Román CA, Ortiz F, Hernández Y. El estrés académico en estudiantes latinoamericanos de la carrera de Medicina. Rev Iberoamericana Edu. [Internet]. 2008 Jul 25; Disponible en: http://rieoei.org/deloslectores/2371Collazo.pdf.

18. Díaz Y. Estrés académico y afrontamiento en estudiantes de Medicina. Rev Hum Med. 2010;10(1).

19. Castaño I, García M, Leguizamón L, etal. Asociación entre el síndrome de estrés asistencial en residentes de medicina interna, el reporte de sus prácticas médicas de cuidados subóptimos y el reporte de los pacientes. Univ Psychol. 2006;5(3):549-62.

20. Estela-Villa LM, Jiménez-Román CR, Landeo-Gutiérrez JS, et al. Prevalencia de síndrome de Burnout en alumnos del séptimo año de medicina de una universidad privada de Lima, Perú. Rev Neuropsiquiatr. 2013;73(4):147-56.

21. da Silva RA, Pinheiro RT, da Silva RA, et al. Functional dyspepsia and depression as an associated factor. Arq Gastroenterol. 2006;43(4):293-8.

22. Talledo-Ulfe L, Buitraigo OD, Filorio Y, et al. Factores asociados a dispepsia funcional en estudiantes de 4 facultades de medicina de Latinoamérica: Estudio multicéntrico. Rev Mex Gastro. 2016; [publicación electrónica antes de impresión].

23. Molano J, Piñeros S, López de Mesa C. Dispepsia y reflujo gastroesofágico en adolescentes escolarizados. Rev Colomb Gastroenterol. 2008;23(1):47-56.

24. Vargas M, Talledo-Ulfe L, Samaniego R, Heredia P, Rodriguez C, Mogollón C, et al. Dispepsia funcional en estudiantes de ocho facultades de medicina peruanas: Influencia de los hábitos nocivos. Acta Gastro Latinoam. 2016; 2016;46:95-101. 